\title{
Markedly increased renal disease mortality and incidence of renal replacement therapy among IDDM patients in Japan in contrast to Allegheny County, Pennsylvania, USA
}

\author{
M. Matsushima ${ }^{1,2}$, N.Tajima ${ }^{2}$, R. E. LaPorte ${ }^{1}$, T. J. Orchard ${ }^{1}$, E. S. Tull ${ }^{1}$, I. F. Gower ${ }^{1}$ and T. Kitagawa ${ }^{3}$ for the \\ Diabetes Epidemiology Research International (DERI) U.S.-Japan Mortality Study Group \\ ${ }^{1}$ Department of Epidemiology, University of Pittsburgh, Pittsburgh, Pennsylvania, USA \\ ${ }^{2}$ Third Department of Internal Medicine, Jikei University School of Medicine, Tokyo, Japan \\ ${ }^{3}$ Medical Research Institute, Nihon University School of Medicine, Tokyo, Japan
}

\begin{abstract}
Summary The aim of this study was to evaluate factors related to the markedly increased risk of dying from diabetic renal disease in Japanese insulin-dependent diabetic patients compared to those in the USA. The study was based on two population-based cohorts consisting of 1374 cases from Japan and 995 cases from Allegheny County, Pennsylvania, USA, who were diagnosed between 1 January 1965 and 31 December 1979. The living status and dialysis experience were determined as of 1 January 1990.

The duration-adjusted renal-failure-related mortality rates in the Japanese cohort and the USA cohort were 277.2 and 130.9 per 100,000 person-years, and the duration-adjusted incidence rates of dialysis were 564.9 and 295.6 per 100,000 person-years, respectively. After adjustment for sex, age at onset, calendar year of onset, and duration of diabetes, individ-
\end{abstract}

uals with insulin-dependent diabetes in the Japanese cohort were still 2.4-fold more likely to receive dialysis compared to those in the USA cohort. Ten of the 36 renal-failure-related deaths in the Japanese cohort had never been treated by dialysis, while all renal-failure-related deaths in the USA cohort had been treated by dialysis. Survival after initiation of dialysis in the Japanese cohort was virtually the same as the USA cohort. These data suggest that a greater frequency of diabetic end-stage renal disease and reduced access to acceptance at dialysis underlie much of the excess of diabetic renal deaths in Japan. [Diabetologia (1995) 38: 236-243]

Key words Insulin-dependent diabetes mellitus, mortality, nephropathy, dialysis, epidemiology.
Before the discovery of insulin, few children survived more than 1 or 2 years after the onset of diabetes mellitus [1]. After the introduction of insulin therapy, life expectancy for insulin-dependent diabetic (IDDM) patients dramatically increased [2]. However, IDDM individuals are still at a markedly increased risk for dying compared with the general population $[2,3]$.

The Diabetes Epidemiology Research International (DERI) mortality study was initiated in 1986

Received: 4 March 1994 and in revised form: 25 August 1994

Corresponding author: Dr. M. Matsushima, 3rd Department of Internal Medicine, Jikei University School of Medicine, 3-25-8 Nishishimbashi Minato-ku, Tokyo 105, Japan

Abbreviations: DERI study, Diabetes Epidemiology Research International study; ESRD, end-stage renal disease; C. I., confidence interval; RRT, renal replacement therapy. to evaluate the geographic differences of IDDM mortality in four diverse population-based cohorts from Finland, Israel, Japan, and Allegheny County, Pa., USA $[4,5]$. The initial results of the DERI study revealed a considerably greater mortality rate in Japan compared to the USA [4]. Much of the excess mortality for the Japanese cohort appeared to be the result of diabetic renal disease. [5] Although IDDM is a rare disease in Japan (incidence rate 1.7/100,000 per year, prevalence rate $10 / 100,000)[6,7]$ compared to European countries and the USA (Fig. 1 [8]), once a child develops diabetes in Japan, their prognosis appears to be considerably poorer, mostly due to a markedly increased risk for renal disease.

The reasons for the excess mortality from renal disease in the Japanese cohort are likely to consist of two components. The first is a difference in risk (incidence) of end-stage renal disease (ESRD). Anoth- 
er is the difference in case-fatality rate whereby once ESRD occurs, individuals are at an increased risk of death. The case-fatality rate can be affected by a number of factors such as quality of medical care, socioeconomic factors, etc. To prevent unnecessary deaths, it is critical to identify the possible factors related to the high renal-disease mortality. Since only renal replacement therapy (RRT) can sustain life for patients suffering from renal failure, RRT may play a critical role in longevity.

The current report evaluates three hypotheses for the excess renal disease mortality in the Japanese cohort, namely a higher incidence of ESRD from diabetic renal disease, poor access/acceptance to dialysis and a poor prognosis in the post-dialysis phase. The first hypothesis was tested by comparing the incidence rates of dialysis therapy and the life-table for dialysis between the two cohorts. The second hypothesis was tested by examining the proportion of cases not treated by dialysis among all deaths in persons with ESRD. The last hypothesis was evaluated by comparing the survival rates in the American and Japanese cohorts after the start of dialysis. Despite the small number of IDDM patients and the even smaller number of IDDM patients with ESRD in Japan, this analysis can provide important insight as to reasons why there is an excess mortality. It can also serve as a model to evaluate reasons for excess mortality in other countries.

\section{Subjects and methods}

Study populations. The inclusion criteria in both cohorts were that each individual was diagnosed as having diabetes before 18 years of age, was placed on insulin at the time of hospital discharge, and was diagnosed between 1 January 1965 and 31 December 1979.

Japan: In 1970 and 1981 [9, 10], two nationwide surveys were conducted by the Childhood Research Committee, the Ministry of Health and Welfare of Japan and 1628 youth-onset patients who had IDDM were identified. After applying the above criteria for classifying IDDM, 1428 cases were eligible. The process for tracing the cases to determine their living status (alive or dead) as of 1 January 1990 comprised several procedures; contacting the physicians using questionnaires, calling family members by telephone, residence registry checks and searching in the list of summer camp participants. The living status was determined as of 1 January 1990 in 1374 of the 1428 eligible subjects $(96.2 \%)$.

Allegheny County (USA): The cohort from the Allegheny County IDDM Registry consists of 1076 patients diagnosed between 1965 and 1979 [11]. It is a population-based registry developed through the review of hospital records and validated by identifying cases through contact with community paediatricians. Follow-up for mortality was undertaken by means of telephone interviews with physicians, patients and family members as well as questionnaires. The living status was determined as of 1 January 1990 in 1009 of the 1076 eligible subjects $(93.8 \%)$.

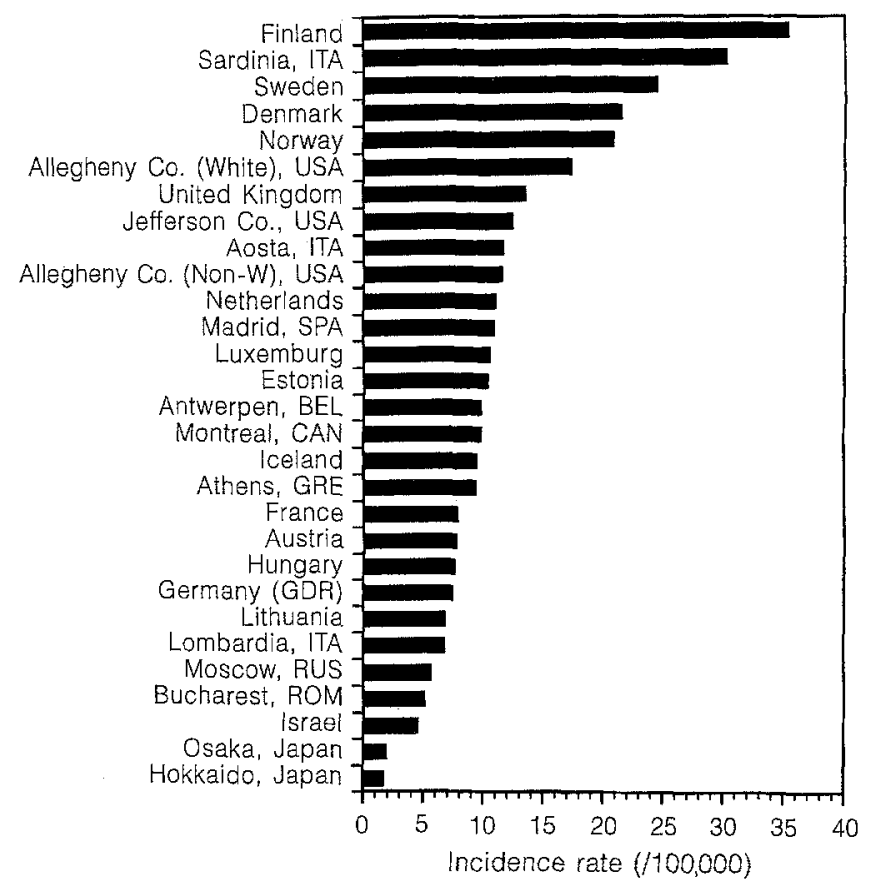

Fig. 1. IDDM incidence rates in various countries (ITA: Italy, USA: the United States, SPA: Spain, BEL: Belgium, CAN: Canada, GRE: Greece, RUS: Russia, ROM: Romania)

There were some differences in the nature of these two cohorts. The Japanese cohort is a prevalence cohort whereas the USA cohort is an incidence cohort. To make the two groups comparable, we excluded from the USA cohort 14 cases who developed IDDM during 1965-1969 and died prior to 1 January 1970, or who developed IDDM during 1970-1979 and died prior to 1 January 1980 . As a result, the USA cohort consisted of 995 eligible subjects. Detailed information about the cohorts has been described elsewhere $[4,5]$.

Classification of renal-failure-related deaths. The process of standardized mortality classification has been described in our previous report [5]. Death certificates, hospital records, coroners' records, and autopsy reports were reviewed by four physicians from the Mortality Classification Committee. The committee determined, 1) the most likely underlying cause of death, 2) the order of importance of all other contributing causes of death, and 3 ) the role of diabetes in causing the death. The causes of death consist of eight categories; acute diabetic complication, diabetic renal disease, infections, accident, suicide, coronary artery disease, other cardiovascular disease, and others. To limit this study to the assessment of the relationship between diabetic ESRD and RRT, we focused on the individuals who died with ESRD and excluded deaths due to other causes even if renal disease was present but ESRD had not developed; for example, deaths from nephrotic syndrome. Renal-failurerelated deaths were defined as deaths with history of ESRD due to diabetic renal disease regardless of the underlying causes of death and the importance of other contributing causes to death. ESRD was defined as being treated by renal dialysis, kidney transplant, or having clinical information including serum creatinine level $\geq 5 \mathrm{mg} / \mathrm{dl}$ and chronic uraemic symptoms and/ or signs. Acute renal failure and renal failure from other causes such as nephritis were excluded from diabetic ESRD.

Determination of the incidence of renal replacement therapy (experience of dialysis). Information concerning the experi- 
ence of RRTwas collected over the same time period as the living status of patients was determined. In Japan, questionnaires were sent to physicians to ask if patients had received dialysis. If information was not available from the physicians, patients or their families were contacted by telephone. Responses were obtained about $94.9 \%$ (1304 of 1374) of the patients. The detailed information on dialysis, for example, which treatment a patient received, haemodialysis or peritoneal dialysis, and the information on renal transplant were not obtained by the questionnaire. In Allegheny County, a short telephone interview with patients or families was undertaken. Responses were obtained from $80.1 \%$ (797 of 995) of the identified cohort. For all deceased cases in the two cohorts, the experience of dialysis was determined primarily through reviewing medical records. Although there was somewhat of a differential response rate between the two cohorts, responders had similar male/female ratio distributions, age at onset and duration of diabetes to those of non-responders in each cohort. Non-responders were excluded from the analyses.

\section{Statistical analysis}

Due to the importance of diabetes duration for the occurrence of chronic complications, renal-failure-related mortality rates and incidence rates of dialysis therapy were adjusted for taking this into consideration. To calculate the duration-adjusted rates, the summation of person-years of duration over both populations in each duration group $(0-4,5-9,10-14,15$-years of duration) was used as the standard population. The duration-adjusted rate can be expressed as a weighted average of the duration-specific rates, where the weights are the proportions of person-years exhibiting each duration group (0-4, 5$9,10-14,15$-years of duration). Person-years were counted from the initial point of follow-up, that is, 1 January 1970 for those who were diagnosed between 1965-1969, and 1 January 1980 for those who were diagnosed between 1970-1979. Ninety-five percent confidence intervals of duration-adjusted rates were estimated using the Poisson distribution [12].

A life table was constructed using product limit estimates in which the log-rank test was used to test the significance of the differences between the cumulative risk curves. The time scale of life-table analyses for renal-failure-related mortality and incidence of dialysis was the follow-up time, and not the duration of diabetes.

Since there were some differences in demographic characteristics between the two cohorts as presented in Table 1 and these characteristics have been previously found to be associated with overall mortality $[13,14]$, they were included in multivariate models to adjust for their independent effect. The Cox proportional hazard model was employed to adjust for sex, age at onset, calendar year of onset, and duration of diabetes. Statistical analyses were made by SAS release 6.08 [15].

\section{Results}

All-cause mortality. Ninety deaths in the Japanese cohort and 58 deaths in the USA cohort were identified as of 1 January 1990 . The duration-adjusted mortality rates of all causes of deaths in the Japanese cohort and the USA cohort up to 20 years of follow-up were 616.3 and 388.5 per 100,000 person-years, respectively.
Table 1. Demographic characteristic of the cohorts

\begin{tabular}{|c|c|c|}
\hline & Japan & USA \\
\hline Sex (Male/Female) $(n)$ & $509 / 770$ & $423 / 371$ \\
\hline $\begin{array}{l}\text { Year of diagnosis }(n) \\
1965-1969 \\
1970-1974 \\
1975-1979\end{array}$ & $\begin{array}{l}250 \\
318 \\
711\end{array}$ & $\begin{array}{l}235 \\
297 \\
269\end{array}$ \\
\hline $\begin{array}{l}\text { Age at onset }(n) \text { (years) } \\
0-4 \\
5-9 \\
10-17\end{array}$ & $\begin{array}{l}311 \\
447 \\
521\end{array}$ & $\begin{array}{r}73 \\
243 \\
485\end{array}$ \\
\hline $\begin{array}{l}\text { Duration of diabetes (person-years) } \\
\begin{array}{l}0-4 \\
5-9 \\
10-14 \\
15-\end{array}\end{array}$ & $\begin{array}{l}2670.3 \\
5697.3 \\
4381.9 \\
2135.8\end{array}$ & $\begin{array}{l}1264.2 \\
3241.8 \\
3375.2 \\
2310.0\end{array}$ \\
\hline $\begin{array}{l}\text { Duration of diabetes at initial point } \\
\text { of follow-up (year, mean } \pm S D \text { ) }\end{array}$ & $3.5 \pm 2.6$ & $4.4 \pm 2.9$ \\
\hline
\end{tabular}

a If an individual developed diabetes on 1 January 1968 and died 1 January 1975 , she/he contributed 3 person-years to the category of $0-4$ year duration from onset and 2 person-years to the category of 5-9 year duration from onset. Therefore, 2 years from 1 January 1968 to 31 December 1969 are not counted as person-years

Renal-failure-related mortality. There were 36 renalfailure-related deaths in the Japanese cohort of 90 deceased cases (40\%), and 17 of $58(29 \%)$ in the USA cohort. The duration-adjusted renal-failure-related mortality rates up to 20 years of follow-up were 277.2 per 100,000 person-years [95\% C.I.: 186.4 $368.1]$ in the Japanese cohort and 130.9 per 100,000 person-years [68.7-193.2] in the USA cohort (Table 2). Figure 2 demonstrates the significant difference in the cumulative risks for deaths between the two cohorts. As shown in Table 1, there were some differences in demographic characteristics between the two cohorts. Surprisingly, although individuals in the Japanese cohort had shorter duration and more recent onset of diabetes compared to those in the USA cohort, both the mortality rate and incidence rate of dialysis in the Japanese cohort were higher than those in the USA cohort as described above. A proportional hazard model revealed that after adjusting for sex, age at onset, calendar year of onset, and duration of diabetes, individuals with IDDM in the Japanese cohort were still over 2.5-fold more likely to die with ESRD compared to those in the USA cohort (Table 3).

Incidence of dialysis. Seventy-two patients in the Japanese cohort and 37 patients in the USA cohort, who had received dialysis therapy, were identified. For those treated by dialysis, age at onset of diabetes and age at initiation of dialysis were $11.6 \pm 3.6$ years (mean $\pm \mathrm{SD}$ ), $28.3 \pm 4.4$ years for the Japanese cohort, and $12.1 \pm 2.8$ years, $29.9 \pm 3.6$ years for the USA cohort. There were no differences in age at on- 
Table 2. Results of mortality and incidence of dialysis

\begin{tabular}{lll}
\hline Mortality & Japan & USA \\
\hline Renal-failure-related deaths/all deaths & $36 / 90(40.0 \%)$ & $17 / 58(29.0 \%)$ \\
Duration-adjusted mortality $(/ 100,000$ person-years) & & \\
Total & $616.3[487.7-744.9]$ & $388.5[287.8-489.2]$ \\
Renal-failure-related & $277.2[186.4-368.1]$ & $130.9[68.7-193.2]$ \\
Incidence of dialysis & & \\
Dialysis cases/all cases & $72 / 1279(5.6 \%)$ & $37 / 794(4.7 \%)$ \\
Duration-adjusted incidence of dialysis (/100,000 person-years) & $564.9[433.8-696.0]$ & $295.6[200.1-391.0]$ \\
\hline
\end{tabular}

$95 \%$ Confidence interval given in parentheses

Table 3. Proportional hazard model: renal-failure-related mortality and incidence of dialysis

\begin{tabular}{|c|c|c|c|c|}
\hline \multirow[t]{2}{*}{ Variables } & \multicolumn{2}{|c|}{ Renal-failure-related mortality } & \multicolumn{2}{|c|}{ Incidence of dialysis } \\
\hline & Relative risk & $95 \%$ C.I. & Relative risk & $95 \%$ C.I. \\
\hline Cohort (Japan/USA) & 2.57 & $1.41-4.68^{b}$ & 2.38 & $1.57-3.60^{\mathrm{b}}$ \\
\hline Age at onset (years) & 1.16 & $1.08-1.25^{\mathrm{b}}$ & 1.21 & $1.15-1.27^{b}$ \\
\hline Calendar year of onset (year) & 0.85 & $0.75-0.97^{\mathrm{a}}$ & 0.98 & $0.88-1.08$ \\
\hline Duration of diabetes at initial point of follow-up (years) & 1.10 & $0.93-1.29$ & 1.36 & $1.21-1.52^{\mathrm{b}}$ \\
\hline
\end{tabular}

Duration of diabetes at an initial point of follow-up: duration of diabetes as of 1 January 1970 for those diagnosed during 1965-1969
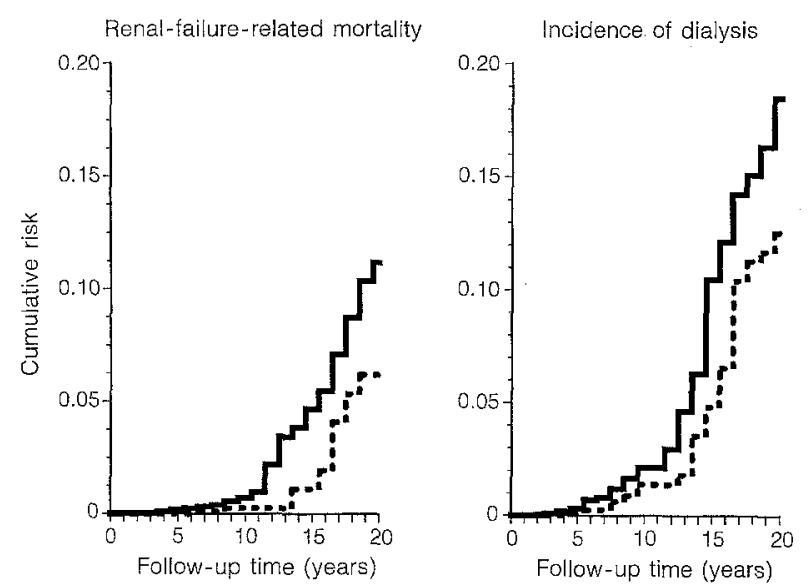

Fig. 2. Cumulative risks for renal-failure-related deaths (left) and dialysis (right) in Japan ( $\boldsymbol{m}$ ) and the USA (ma). Significant differences were detected in both cumulative mortality and incidence of dialysis between Japan and the USA at $p<0.05$

set of diabetes and age at initiation of dialysis between deceased patients and those who were alive. The duration-adjusted incidence rates of dialysis in the Japanese cohort and the USA cohort up to 20 years of follow-up were 564.9 per 100,000 personyears [95\% C.I.: 433.8-696.0] and 295.6 [200.1391.0], respectively. A life-table analysis for incidence of dialysis is also presented in Figure 2. After adjusting for sex, age at onset, calendar year of onset, and duration of diabetes by proportional hazard model, individuals in the Japanese cohort were ap- duration of diabetes as of 1 January 1980 for those diagnosed during 1970-1979

a $p<0.05$

b $p<0.01$

proximately 2.4-times more likely to receive RRT than those in the USA (Table 3 ). These results probably indicate that considerably more patients develop ESRD among those who have IDDM in Japan than in the USA. This pattern of difference between the two cohorts was quite similar to that shown for renal-failure-related mortality.

Duration-specific rates for renal-failure-related mortality and incidence of dialysis. As shown in Figure 3, individuals in the Japanese cohort had higher rates over all groups of duration categories with regard to both renal-failure-related mortality and incidence of dialysis. Of interest is that the patients in the Japanese cohort potentially develop ESRD even earlier than those in the USA cohort in terms of duration of diabetes.

Proportion of non-dialysis cases among renal-failurerelated deaths and its time trend. To evaluate the access to acceptance of dialysis therapy, the current study compared the proportion of those without RRT among renal-failure-related deaths between Japan and the USA. In the USA cohort, all renal-failure-related deaths other than one patient for whom information on dialysis was not available, had been treated by dialysis $(16 / 16)$ and transplantation $(5 / 16)$. In contrast, 10 of the 36 patients who died with ESRD $(27.8 \%)$ in the Japanese cohort had never been treated by dialysis. Among 36 renal-relateddeaths in Japan, none had received a transplant. Among those who died with ESRD prior to 1985 in 
Japan, $30 \%(6 / 20)$ had never been treated by dialysis. Of interest is that even in the late 1980's (1985-1989) when dialysis facilities were more widely available in Japan [16], still 25\% (4/16) of cases who died had never received dialysis therapy.

\section{Life-table analysis after the initiation of dialysis}

To examine the post-dialysis phase of diabetic ESRD in the two cohorts, a life-table analysis was conducted (Fig.4). This figure indicates that survival function curves after the initiation of dialysis were not significantly different between Japan and the USA. Table 4 also indicates no significant difference in the postdialysis mortality between the two cohorts after adjusting for confounding factors. Thus, once the RRT was initiated, those with ESRD in Japan did not appear to be at a greater risk for death compared to those in the USA.

\section{Discussion}

One of the major concerns for the care of diabetes is how to prevent chronic complications. Diabetic renal disease is a major killer of youth-onset patients who have IDDM in Japan $[5,17]$. We have seen that patients in the Japanese cohort have a considerably higher renal-failure-related mortality risk than those in the USA cohort. To prevent unnecessary deaths, it is critical to identify why Japanese patients are more likely to die with ESRD.

First, the difference in dialysis experience between responders and non-responders should be examined to consider the selection bias. High response rates over $80 \%$ and few differences in the pattern between incidence of dialysis and renal-failure-related mortality suggest little selection bias. Second, we must examine whether other causes of death, in particular cardiovascular disease, were responsible for the difference in renal-failure-related mortality to consider competing risks. Since our cohorts are still quite young, cardiovascular disease was rare and little difference was observed in the cardiovascular mortality between the two cohorts (duration-adjusted cardiovascular mortality, Japan: 52.0 [95\% C.I. 13.2-90.1] per 100,000 person-years, USA: 76.3 $[33.0-120.0], p>0.05)$. Thus, the difference in cardiovascular mortality between the two cohorts cannot account for the difference in renal-failure-related mortality. All other cause-specific mortality rates in the Japanese cohort were higher than those in the USA cohort. The difference in renal-failure-related mortality is not likely to be the result of renal disease deaths being assigned a different cause of death.

Two major factors determine the mortality rate; the incidence of ESRD and the case fatality rate in

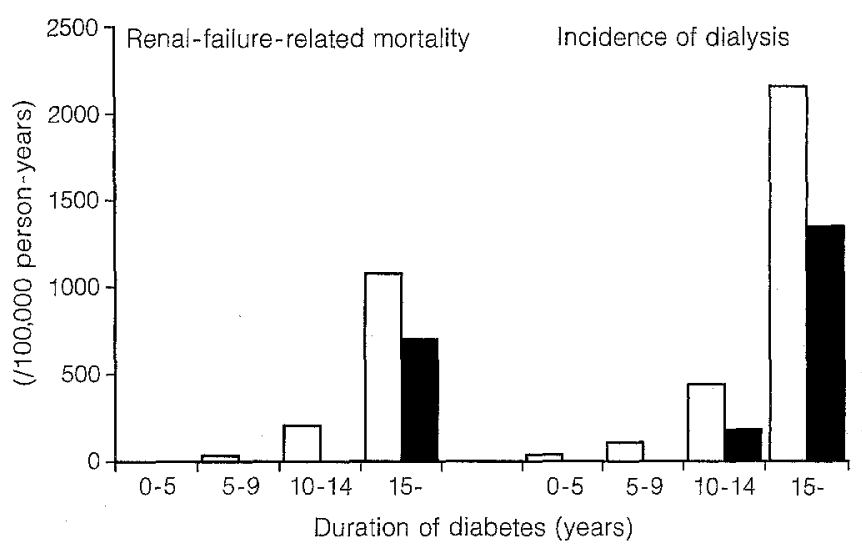

Fig. 3. Duration-specific rates of renal-failure-related mortality and incidence of dialysis in Japan ( $\square$ ) and the USA ( $\mathbf{a})$

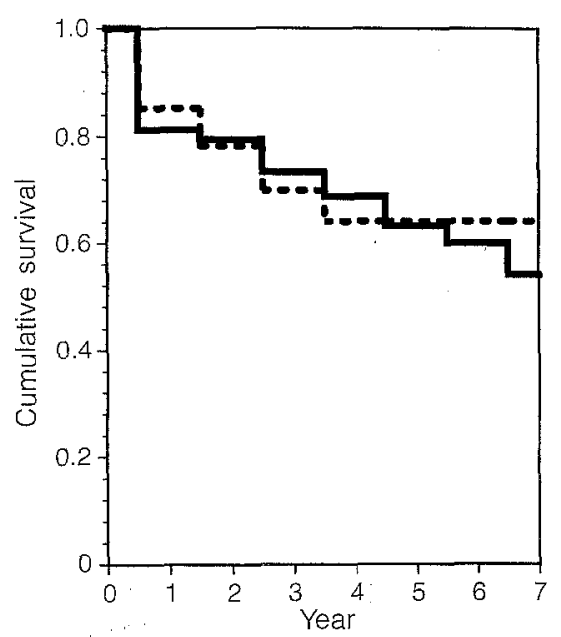

Fig.4. Cumulative survival functions after the initiation of dialysis in Japan (m) and the USA (ma)

Table 4. Proportional hazard model: survival after initiation of dialysis

\begin{tabular}{llll}
\hline Variables & Relative risk & $95 \%$ C.I. & $p$ value \\
\hline Cohort (Japan/USA) & 1.01 & $0.46-2.23$ & 0.98 \\
Sex (female/male) & 1.66 & $0.74-3.74$ & 0.22 \\
Age at onset (year) & 1.02 & $0.90-1.14$ & 0.80 \\
Calendar year of onset & 0.84 & $0.72-0.99$ & 0.04 \\
$\begin{array}{l}\text { Duration of diabetes } \\
\text { at initiation of dialysis }\end{array}$ & 0.93 & $0.82-1.05$ & 0.24 \\
\hline
\end{tabular}

the patients with ESRD. The current study estimated the risk for ESRD by evaluating the incidence of dialysis therapy. Overall there appeared to be considerable differences in the risk for developing ESRD between the two groups, as the incidence of dialysis therapy in the Japanese cohort was 2.4-fold greater compared to the USA cohort. We must be cautious, however, as this interpretation is based on an indirect measure of the incidence of ESRD and there is likely to be some selection bias involved (e.g., referral bias) [18]. The true proportion of diabetic pa- 
tients with ESRD are certainly underestimated in both groups. However, it appears that the incidence of ESRD is likely to be much greater in the Japanese cohort than the USA cohort, since the proportion of ESRD in Japan should be more underestimated than in the USA, due to poor access to acceptance of dialysis facilities in Japan as described below.

Another explanation for the excess renal-failurerelated mortality in the Japanese cohort is poor access/acceptance to RRT. The current report demonstrates that all renal-failure-related deaths in the USA cohort had received RRT that consisted of initial dialysis and kidney transplantation. In contrast, almost one-third of those who died with ESRD in the Japanese cohort had never been treated by dialysis. A previous report demonstrated that the acceptance rate for RRT among the general Japanese population was similar to that in the USA (acceptance rate: Japan 137, USA 151/million population/year) [19]. In that study, only $22 \%$ of patients accepted for RRT in Japan had diabetes, while $30 \%$ in the USA had diabetes. If the data from the present study could be extrapolated to the general Japanese IDDM population with ESRD, this lower proportion with respect to the USA could explain the grounds of a higher proportion of patients not accepted for RRT in Japan. To estimate how much the major differences in renal-failure-related mortality could be attributed to the poor access/acceptance of dialysis therapy, we determined what would have happened if the 10 non-dialysis renal-failure-related deaths in the Japanese cohort had been excluded from the analysis. In this case the duration-adjusted mortality could have decreased from 277.2 to 204.5 (per 100,000 person-years); however, this is still much greater than the USA rate $(130.9$ per 100,000$)$, suggesting that at most $50 \%[=(277.2-204.5) /(277.2-$ 130.9)] of the excess renal-failure-related mortality in the Japanese cohort compared to the USA cohort likely resulted from lack of use of dialysis.

Another concern is whether the Japanese cohort differs from the USA cohort as to the prognosis after the initiation of dialysis. A major difference in RRT between the two cohorts is a kidney transplant. Unfortunately, in the current study, we have no information about transplantation among those who did not die in the Japanese cohort. Although the acceptance rate of kidney transplant in the general population of Japan is much lower than that of the USA [16, 19], a life-table analysis suggested that there was no difference between the Japanese cohort and the USA cohort with regard to longevity after dialysis. These results indicated that the post-dialysis factors appear to contribute little to the differences in renalfailure-related mortality between the two cohorts. However, it may be difficult to generalize about this result due to the small sample size of patients with ESRD and the lack of information on type of RRT.
According to the previous report, only $4.4 \%$ of Japanese with ESRD receive peritoneal dialysis compared to $12.7 \%$ of Americans with ESRD [20]. Further investigation is needed to evaluate the post-dialysis prognosis, in particular, the detailed information on modalities for RRT, for example, haemodialysis, peritoneal dialysis or transplantation among IDDM patients with ESRD.

The present study revealed at least two potential reasons for the marked excess mortality from ESRD in the Japanese cohort; a greater incidence of ESRD measured as incidence rate of dialysis therapy and a possible higher case-fatality rate due to poor access/ acceptance of dialysis therapy. However, it remains essential to determine the factors which contribute to the difference in incidence of ESRD. In terms of diabetes care, poor glycaemic control could be one major factor since insulin self-injection was not allowed by law until 1980 in Japan and there were children with IDDM who could not receive insulin injections on Sunday. However, there is no data available for comparison of glycaemic control between Japan and the USA. Other environmental factors such as treatment for hypertension, cigarette smoking [21], high protein diet [22], and salt intake [17] can have an impact on the difference in frequency of ESRD. In terms of host susceptibility to ESRD, there appear to be many related factors; for example, 1) differences in genetic susceptibility to hypertension, 2) differences in genetic susceptibility to diabetic renal disease, 3) genetic differences in the rate for progression of renal disease to ESRD [22-28]. The current results showing that Japanese patients with IDDM may have an earlier onset of ESRD suggests that at least the rate of progression to ESRD may be higher among Japanese than Americans. We cannot, however, determine which genetic or environmental factors have an impact on the development of ESRD in the current study. Moreover, we have only addressed the incidence of diabetic ESRD, and not the incidence of diabetic renal disease itself. Clearly, additional research is needed to determine whether the higher incidence of ESRD in the Japanese cohort reflects a higher incidence of diabetic renal disease or a more rapid progression to ESRD.

One of the factors affecting fatality rates may be the accessibility/acceptance of RRT. A long distance between the place of residence and the dialysis clinic may be a problem. Our previous report suggested that patients who live in rural areas with fewer facilities may have a reduced access to RRT [29]. However, this can only be a partial explanation, as during the 1980's there was a rapid increase in the number of dialysis facilities [16]. One might have expected a corresponding reduction of the percentage of deceased cases who had never been treated by dialysis in the late 1980's. However, this was not the case. Another factor associated with access to dialysis therapy 
may be the selection of patients for RRT by physicians. The difference in acceptance of patients with diabetes of RRT between Japan and the USA may be partially related to the higher mortality in the Japanese cohort [19]. Patient compliance is also important. The difference in socioeconomic status or cultural environmental that leads to lower compliance is likely to contribute to the higher mortality [30].

In conclusion, there is a clear need to better understand the aetiology of diabetic renal disease and ESRD to reduce the excess incidence in Japan, as well as a need to overcome the poorer access to acceptance of dialysis in Japan. These findings will help provide an answer to the question of how to prevent unnecessary deaths due to diabetic ESRD in not only Japan, but Europe and other countries around the world. Moreover, it remains essential to determine the incidence and prevalence of diabetic renal disease and ESRD among individuals with IDDM in a standardized method in Japan and other countries. This is currently being developed as part of the World Health Organization Diamond project [31].

Acknowledgements. Research for this work was supported by National Institute of Health Grant DK-35905 (USA), and Ministry of Health Scientific Grant (Japan).

DERI US-Japan Mortality Study Group; Allegheny County, Pa., USA: R.E. LaPorte, T. J. Orchard, E. S. Tull, I.F. Gower, J.F. Payne, E.J. Barinas, T.J. Songer, R. J. Vergona. Japan: T.Kitagawa, N.Tajima, M.Aono, S. Aono, I. Hibi, Y.Ikeda, G. Isshiki, M. Kawamura, K. Kida, Y.Kohno, H.Maruyama, M.Maruyama, M.Matsushima, N.Matsuura, E.Miki, E. Mimura, R. Nishimura, K. Shimizu, A. Takeda, T. Toyota.

\section{References}

1. Krolewski A, Warram J, Christlieb A (1985) Onset, course, complication and prognosis of diabetes mellitus. In: Marble A, Krall LP, Bradley RF, Christlieb AR, Soeldner JS (eds) Joslin's Diabetes Mellitus. Lea \& Febige, Philadelphia, PA, pp. 251-277

2. Borch-Johnsen K (1989) The prognosis of insulin-dependent diabetes mellitus. Danish Med Bull 36: 336-348

3. Panzram G (1984) Epidemiologic data on excess mortality and life expectancy in insulin-dependent diabetes mellitus. Crit Rev Exp Clin Endocrinol 82: 93-100

4. Diabetes Epidemiology Research International Group (1991) Major cross country differences in risk of dying for people with IDDM. Diabetes Care 14: 49-54

5. Diabetes Epidemiology Research International Group (1991) International evaluation of cause-specific mortality and IDDM. Diabetes Care 14: 55-60

6. Matsuura N, Fukushima N, Fujita H et al. (1983) Epidemiologic survey of juvenile onset insulin dependent diabetes mellitus (IDDM) in Hokkaido, Japan. Tohoku J Exp Med 141 [Suppl]: 181-189

7. Kuzuya T, Ito C, Sasaki A et al. (1992) Prevalence and incidence of diabetes in Japanese people compiled from the literature - A report of the epidemiology data committee, the Japan Diabetes Society. J Japan Diab Soc 35: 173-194

8. Karvonen M, Tuomilehto J, Libman I et al. (1993) A review of the recent epidemiological data on incidence of type 1 (insulin-dependent) diabetes mellitus worldwide. Diabetologia 36: 883-892

9. Miki E, Maruyama H (1972) Juvenile-onset diabetes mellitus in Japan - the results of the first nation-wide survey. J Jpn Diab Soc 15: 38-42

10. Hibi I, Tanae A, Maruyama H et al. (1981) Juvenile-onset type diabetes in Japan: current status. Report of the Childhood Diabetes Research Committee. the Ministry of Health and Welfare, Japan, 1980-1981

11. Lestradet H, Papoz L, DeMenibus CL et al. (1981) Longterm study of mortality and vascular complications of juvenile-onset diabetes. Diabetes 35: 175-179

12. Boyle P, Parkin DM (1991) Statistical methods for registries. In: Jensen OM, Parkin DM, MacLennan R, Muir CS, Skeet RG (eds) Cancer registration: principles and methods. International Agency for Research on Cancer, IARC Scientific Publications No. 95, Lyon, France, pp. 126-158

13. Dorman JS, LaPorte RE, Kuller LH et al. (1984) The Pittsburgh insulin-dependent diabetes mellitus (IDDM) morbidity and mortality study. Mortality results. Diabetes 33: 271-276

14. Kostraba JN, Dorman JS, LaPorte RE et al. (1991) The investigation of age at onset as a risk factor for mortality in persons with insulin-dependent diabetes mellitus using Cox proportional hazard models. Am J Epidemiol 133: $67-72$

15. SAS Institute Inc. (1990) SAS/STAT User's Guide, Version 6. SAS Institute Inc., Cary, NC

16. Kishimoto T (1990) Present status of ESRD treatment in Japan. In: Klinkmann H, Smeby LC (eds). Terminal renal failure: therapeutic problems, possibilities, and potentials. Contrib Nephrol, Vol 78. Basel, Karger, pp. 178-180

17. Patrick SL, Tajima N, LaPorte RE, Kitagawa T (1992) A comparison of renal disease mortality among individuals with insulin-dependent diabetes mellitus (IDDM) in Japan and Allegheny County, Pa., USA. J Jpn Diab Soc 35: 993 1000

18. Catalano C, Marshall SM (1992) Epidemiology of endstage renal disease in patients with diabetes mellitus: from the dark ages to the middle ages. Nephrol Dial Transplant 7: $181-190$

19. Hull AR, Parker TF (1990) Proceedings from the morbidity, mortality and prescription of dialysis symposium, Dallas, Texas, September 15 to 17, 1989. Am J Kidney Dis 15: 375-383

20. United States Renal Data System Coordinating Center (1993) International Comparison of ESRD Therapy. USRDS 1993 Annual Data Report

21. Stegmayr B, Lithner F (1987) Tobacco and end stage diabetic nephropathy. BMJ 295: 581-582

22. Brenner BM, Meyer TW, Hostetter TH (1982) Dietary protein intake and the progressive nature of kidney disease: the role of the hemodynamically mediated glomerular injury in the pathogenesis of progressive glomerular sclerosis in aging, renal ablation, and intrinsic renal disease. $\mathrm{N}$ Engl J Med 307: 652-659

23. Krolewski AS, Canessa M, Warram JH et al. (1988) Predisposition to hypertension and susceptibility to renal disease in insulin-dependent diabetes mellitus. $N$ Engl J Med 318: 140-145

24. Barzilay J, Warram JH, Bak M, Laffel LM, Canessa M, Krolewski AS (1992) Predisposition to hypertension: risk factor for nephropathy and hypertension in IDDM. Kidney Int 41: 723-730

25. Smith SR, Svetkey LP, Dennis VW (1991) Racial differences in the incidence and progression of renal disease. Kidney Int 40: 815-822 
26. Pettitt DJ, Saad MF (1984) Inheritance of predisposition to renal insufficiency in diabetic men. Diabetes 37 (Suppl): $51 \mathrm{~A}$ (Abstract)

27. Seaquist ER, Goetz FC, Rich S, Barbosa J (1989) Familial clustering of diabetic kidney disease. Evidence for genetic susceptibility to diabetic nephropathy. N Engl J Med 320: 1161-1165

28. Jones SL, Viberti GC (1989) Hypertension and microalbuminuria as predictors of diabetic nephropathy. Diabete Metab 15: 327-332
29. Kasuga N, Tajima N, Matsushima M, Kitagawa T, LaPorte RE and DERI group (1992) Relationship between mortality status of children with IDDM and regional characteristics. J Japan Diab Soc 35: 391-396

30. Matsushima M, Tajima N, Kitagawa T (1993) Social and economic risk factors for IDDM deaths in Japan: a population based case control study. Diabetes 42 (Suppl 1): $200 \mathrm{~A}$ (Abstract)

31. WHO Diamond Project Group (1990) WHO Multinational Project for Childhood Diabetes. Diabetes Care 13: 10621068 